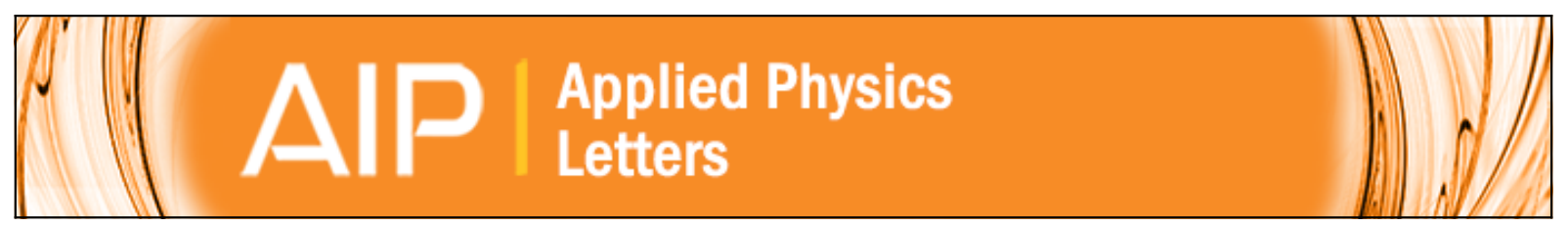

\title{
Coherent detection of metal-metal terahertz quantum cascade lasers with improved emission characteristics
}

Anthony Brewer, Joshua R. Freeman, Pierrick Cavalié, Jean Maysonnave, Jérôme Tignon, Sukhdeep S. Dhillon, Harvey E. Beere, and David A. Ritchie

Citation: Applied Physics Letters 104, 081107 (2014); doi: 10.1063/1.4866267

View online: http://dx.doi.org/10.1063/1.4866267

View Table of Contents: http://scitation.aip.org/content/aip/journal/apl/104/8?ver=pdfcov

Published by the AIP Publishing

\section{Articles you may be interested in}

High power terahertz quantum cascade lasers with symmetric wafer bonded active regions

Appl. Phys. Lett. 103, 171113 (2013); 10.1063/1.4826943

Beam and phase distributions of a terahertz quantum cascade wire laser

Appl. Phys. Lett. 102, 111113 (2013); 10.1063/1.4798250

Improved terahertz quantum cascade laser with variable height barriers

J. Appl. Phys. 111, 103106 (2012); 10.1063/1.4719071

Design concepts of terahertz quantum cascade lasers: Proposal for terahertz laser efficiency improvements Appl. Phys. Lett. 97, 261106 (2010); 10.1063/1.3524197

Electrically tunable terahertz quantum-cascade laser with a heterogeneous active region Appl. Phys. Lett. 95, 181101 (2009); 10.1063/1.3253714

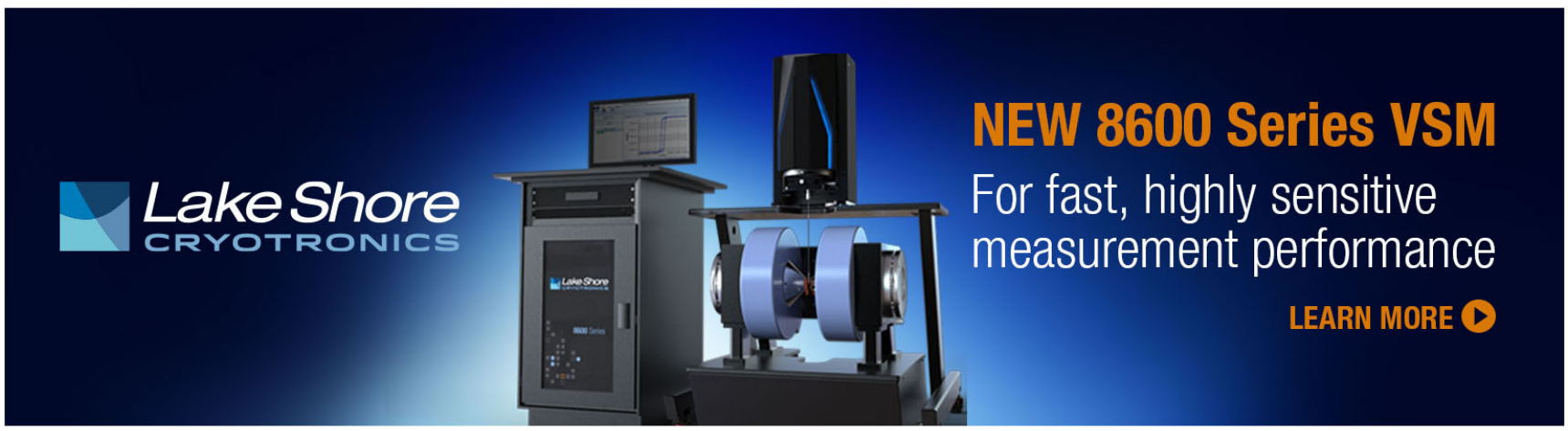




\title{
Coherent detection of metal-metal terahertz quantum cascade lasers with improved emission characteristics
}

\author{
Anthony Brewer, ${ }^{1}$ Joshua R. Freeman, ${ }^{2, a)}$ Pierrick Cavalié, ${ }^{2}$ Jean Maysonnave, ${ }^{2}$ \\ Jérôme Tignon, ${ }^{2}$ Sukhdeep S. Dhillon, ${ }^{2}$ Harvey E. Beere, ${ }^{1}$ and David A. Ritchie ${ }^{1}$ \\ ${ }^{1}$ Semiconductor Physics, Cavendish Laboratory, University of Cambridge, JJ Thomson Avenue, \\ Cambridge CB3 OHE, United Kingdom \\ ${ }^{2}$ Laboratoire Pierre Aigrain, Ecole Normale Supérieure, CNRS (UMR 8551), Université P. et M. Curie, \\ Université D. Diderot, 75231 Paris Cedex 05, France
}

(Received 7 November 2013; accepted 4 February 2014; published online 25 February 2014)

\begin{abstract}
Coherent detection of emission from quantum cascade lasers with metal-metal waveguides is demonstrated through free-space coupling of a $\mathrm{THz}$ pulse to the sub-wavelength waveguide. We implement a simple, monolithic planar horn antenna design on the metal-metal waveguide that reduces the impedance mis-match to the waveguide. The resulting devices show up to 10 times more directed output power than conventional metal-metal waveguides. This enhanced coupling to free-space allows a more efficient injection of broad-band $\mathrm{THz}$ pulses into the waveguide. Through this, we are able to seed the laser emission and coherently detect the laser emission by electro-optic sampling. (C) 2014 AIP Publishing LLC. [http://dx.doi.org/10.1063/1.4866267]
\end{abstract}

Terahertz quantum cascade lasers (QCLs) are a compact semiconductor source of laser radiation operating in the $\mathrm{THz}$ range. These devices show output powers of up to 100's of $\mathrm{mWs}^{1}$ and operating temperatures up to $199 \mathrm{~K}^{2}$ In addition, the emission frequency of these uni-polar lasers is dictated by the active region design, so the emission frequency is more flexible than that of diode lasers which are constrained by the bandgap of the material. Because these lasers operate in the relatively under exploited $\mathrm{THz}$ region of the electromagnetic spectrum, there is significant potential for these devices to find applications in chemical sensing, security applications, biomedical imaging, and characterization of art works, to name just a few. ${ }^{3}$

A recent development is the coherent detection of $\mathrm{THz}$ QCLs. ${ }^{4}$ This could be an important application for QCLs as the coherent detection allows one access to both the timedomain and frequency-domain information in a single measurement. It also allows QCLs to be integrated with $\mathrm{THz}$ time-domain spectroscopy (TDS) systems, which could allow for experiments using a powerful, narrow-band QCL pump and a broadband $\mathrm{THz}$ probe. Until now, this technique for coherent detection has only been used with QCLs that use a single plasmon waveguide. This does not allow the technique to take full advantage of the highest performance $\mathrm{THz} \mathrm{QCLs}$, in terms of temperature and threshold current, that are obtained with a metal-metal (MM) waveguide. The major factor for why MM QCLs have not been used is the difficulty of injecting a $\mathrm{THz}$ seed pulse into the small modevolume of the sub-wavelength MM waveguide. This small mode volume and high mode index also means that MM QCLs tend to have poor output powers and highly divergent beams. 5

There have been attempts to improve the output characteristics of MM QCLs by making use of 2nd and 3rd order distributed feedback techniques ${ }^{6,7}$ and $2 \mathrm{D}$ photonic crystals. ${ }^{8}$ While these methods have increased output power and

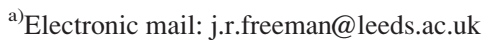

reduced beam divergence, they are intrinsically frequency selective and thus not suitable for all applications. In particular, the frequency selective coupling is not suitable for coupling broadband pulses into the QCL cavity, particularly useful for injection seeding, mode locking of QCLs, or using QCLs as amplifiers in THz TDS systems.

A well-known method for free-space coupling to waveguides at radio frequencies is tapering to create horn antennas; these create an impedance gradient that reduces reflections at the waveguide-free-space interface. These methods have already been used with MM QCL waveguides to produce vertical tapering ${ }^{9}$ and metallic micro-transverse-electromagnetic horn antennas. ${ }^{10,11}$ However, the methods so far demonstrated suffer from complicated fabrication or challenging optical alignment. There have been demonstrations of generating $\mathrm{THz}$ pulses on the facet of MM QCLs, ${ }^{12}$ however, this is not an efficient process and also reduces the flexibility of the experiment. Furthermore, none of these techniques have shown the possibility of seeding or coherent detection of the QCL.

In this work, we present fabrication and characterization of MM QCL waveguides with enhanced broadband freespace-waveguide coupling. The fabrication of these structures is simplified compared to the previous attempts to integrate horn-antenna-like couplers with MM QCLs. Furthermore, we show that these devices are well suited to optical injection seeding, which allows for the coherent detection of the emitted radiation by electro-optic sampling.

We first investigate the far-field radiation pattern and power of MM waveguides that have been modified by the removal of a V-shaped section from both ends of the top metal contact, the design is shown in Figure 1. We refer to these $\mathrm{V}$-shaped areas as planar horn antennas (PHAs). Because in MM QCL waveguides, the confinement is provided by the metal layers above and below the active region, removing some of this metal layer will reduce the confinement and hence the mode index. We bring about a gradual change in the mode confinement by using a taper from full metal 
coverage to a bare ridge, using a similar design philosophy as a horn antenna. These PHAs are more robust than previous attempts to fabricate "3-D" horn antennas on QCLs, and this monolithic geometry also has the advantage of simplicity. Because we retain the planar geometry of the structure, this design could be easily fabricated by standard photolithographic techniques. In this work, we use a focused ion beam (FIB $)^{13}$ to mill the PHAs. This allows us to systematically optimize the extracted power and far-field emission by an iterative fabrication process.

We first investigate the effect the PHA design has on the cavity with the help of a commercial 3D FDTD package (CST-Microwave Studio). Schematics of the simulated device are shown in Figures 1(a) and 1(b), with a 2D slice of the solved solution superimposed onto the $3 \mathrm{D}$ geometries. The modeled THz QCL is $3 \mathrm{~mm}$ in length, $80 \mu \mathrm{m}$ wide, and with a $\mathrm{V}$-shaped section removed from the top metallization of the waveguide to form the PHA. The length of the taper from the start to the facet is $250 \mu \mathrm{m}$ and this is the dimension that we shall use to characterize these PHAs. In the simulation, a symmetry plane is assumed half way so the simulation intensity is reduced, and the Fabry-Pérot cavity is bounded by a $3 \mathrm{D}$ absorbing boundary condition to act as a boundary to free space and produce minimal reflection. A $100 \mu \mathrm{m}$ distance was set for the boundary condition so as not to be within the reactive near-field of the model. For simplicity, the top and bottom doped layers of the QCL were neglected and Au layers treated as Perfect Electric Conductors (PECs). Simulations have been conducted to calculate the reflection and transmission coefficients for several PHA lengths from 0 (no PHA) to $300 \mu \mathrm{m}$ for frequencies in the range of 2.6 $\mathrm{THz}-3.0 \mathrm{THz}$.

Our simulations show that as the PHA length is increased the reflectivity of the facets decreases. For a PHA length of $250 \mu \mathrm{m}$, this reduction in facet reflectivity is around $30 \%$, from $\mathrm{R}=0.8$ for the unperturbed facet to $\mathrm{R}=0.55$ with the PHA. Furthermore, the response is largely insensitive to the frequency of the excitation.

Devices were fabricated from a $2.9 \mathrm{THz}$ bound-to-continuum design, ${ }^{14}$ consisting of 90 repeat periods of a $\mathrm{GaAs} / \mathrm{Al}_{0.15} \mathrm{Ga}_{0.85} \mathrm{As}$ heterostructure. The wafer, grown by molecular beam epitaxy, was bonded to a highly doped $\mathrm{GaAs}$ handle wafer with a $\mathrm{Au}-\mathrm{Au}$ thermo-compression bond. The original substrate was then removed with a combination

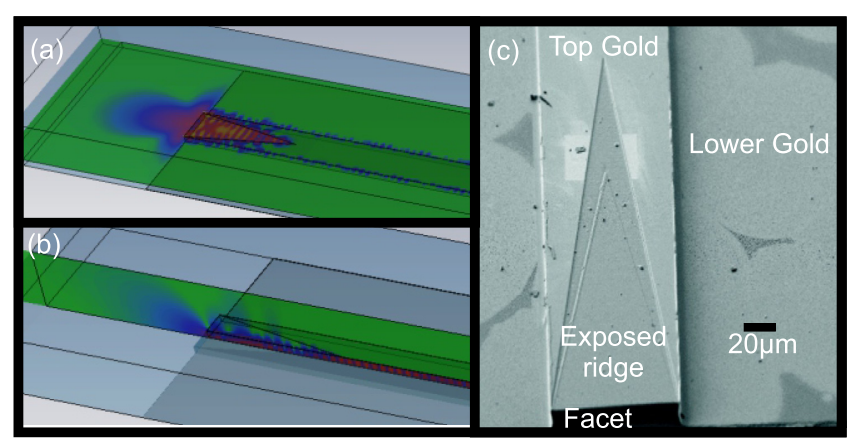

FIG. 1. (a) and (b) Simulations of the PHA designs showing the coupling of the THz field to free-space at the facet. Top and side views of the QCL ridge are shown in (a) and (b), respectively. (c) SEM micrograph of one QCL facet with the PHA defined by FIB, the previous mill is visible. of mechanical polishing and selective wet etching. Laser ridges were dry plasma etched to form ridge waveguides $80 \mu \mathrm{m}$ wide and $3.0 \mathrm{~mm}$ in length. These were soldered to copper sub-mounts and mounted on the cold finger of a continuous-flow liquid helium cryostat.

Our iterative approach to fabrication of the PHAs means that the devices are characterized electrically and for far-field power distribution before any FIB milling takes place and then after each FIB milling step. The FIB provides a convenient micro machining tool for fabrication, with excellent control over the removal of metal from the waveguide (Figure 1(c)), allowing quick prototyping of photonic structures without the cost and time of fabricating masks for UV photolithography. The milling depth was carefully controlled by calibration on sacrificial devices, to ensure that the metal layer and highly doped top layer were removed but the active region was not milled. Removing the highly doped contact layer reduces the loss and confinement of this semi-metallic layer.

Current-voltage (IV) measurements are shown in Figure 2(a) for an "as-cleaved" device, before the PHA is milled, and the same device after progressively longer PHAs are defined in the top metal layer on both ends of the device. The experimental results were acquired under pulsed operation at $10 \mathrm{kHz}$ and $20 \%$ duty cycle. When the device is below threshold, there is small difference of around 5\% between the IVs for the as-cleaved and $275 \mu \mathrm{m}$ device indicating that we have not significantly affected the electrical characteristics of the device. When the device is above threshold, the differences are significant. We note that (i) the threshold increases, shown in Figure 2(b), a sign of a reduced figure of merit $\left(\mathrm{FOM}=\frac{\text { Overlap }}{\text { Loss }}\right)$ for the waveguide; (ii) the optically assisted transport (indicated by the change of slope in the IV at threshold) reduces, suggesting a reduction of optical intensity in the active region; and (iii) the maximum current decreases (the current at cessation of laser action), while the maximum voltage remains the same. This again indicates a reduced optical intensity in the active region. Taken together these observations indicate that the reflectivity of the facets has reduced and more power has been coupled into freespace, with the obvious side-effect that the total losses for the waveguide have increased and the intra-cavity intensity has decreased.

From the increase in threshold current and by assuming that the overlap and waveguide losses remain the same when the PHA is implemented, we may estimate the increase in mirror loss associated with the PHA. In Ref. 15, Jukam et al.
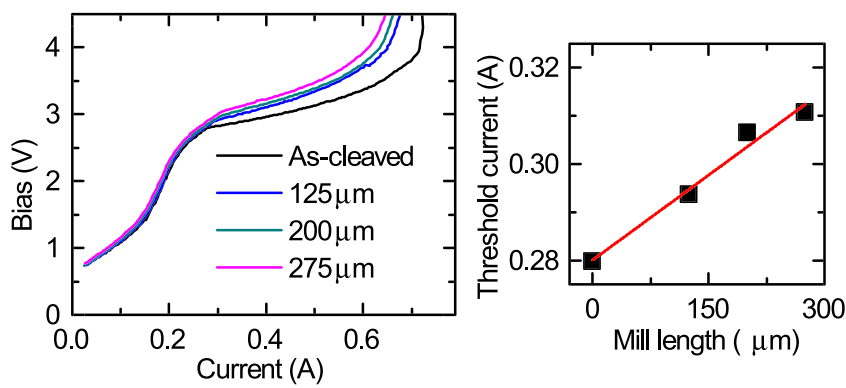

FIG. 2. (a) Pulsed IV plot for the as-cleaved laser, PHA lengths of 125, 200, and $275 \mu \mathrm{m}$ (10 K heat sink temperature). (b) The threshold current for different mill lengths. 
measured the modal gain for the same active region as used in this work. The modal gain around threshold is approximately linear with current and can be written as

$$
\Gamma_{\mathrm{SP}} G=g\left(J-J_{0}\right)
$$

where $\Gamma_{\mathrm{SP}} \simeq 0.3$ is the overlap for the single plasmon waveguide (calculated from simulations), $G$ is the material gain, $g=0.11 \mathrm{~cm} / \mathrm{A}$ is the gain coefficient, $J$ is the current density, and $J_{0}=42 \mathrm{~A} / \mathrm{cm}^{2}$ is the leakage current density. Now, by equating the gain and loss at threshold and assuming that the waveguide loss does not change, we find that the change in threshold current density of the metal-metal waveguide due to a change in mirror losses is

$$
\Delta J_{\mathrm{th}}=\frac{\Gamma_{\mathrm{SP}}}{\Gamma_{\mathrm{MM}}} \frac{\Delta \alpha_{\mathrm{m}}}{g},
$$

where $\Gamma_{\mathrm{MM}} \simeq 1$ is the optical overlap with the active region for a metal-metal waveguide and $\alpha_{m}=-\frac{1}{L} \ln R$ is the mirror loss for a cavity of length $L$ where each facet has a reflectivity $R$. Taking the change in threshold current seen in Figure 2(b), we find that between the un-milled device and the $275 \mu \mathrm{m}$ PHA, the mirror losses increase by $4.8 \mathrm{~cm}^{-1}$. The calculation can be extended to take into account the reduction in current below threshold of 5\%, which implies that the PHA regions are biased at around half the value of the main active region. Taking this imperfect current spreading in the PHA region into account, we find an increase in mirror losses of $4.4 \mathrm{~cm}^{-1}$. Given the calculated value for the unperturbed facet of $R=0.8$, this implies the facet reflectivies has dropped to $R=0.21$, somewhat more than the calculated drop. However, we expect this value to be a lower-bound because the optical overlap with the active region may also decrease when the PHA is added due to mode leakage above the exposed active region.

In addition to the LIV characterization, we have also measured the far-field emission of the MM devices by mounting a Golay cell on an arm attached to a rotation stage with the QCL on the axis of rotation. For these measurements, the device was electrically chopped at $15 \mathrm{~Hz}$ in addition to $10 \mathrm{kHz}$ pulses to enable lock-in detection for the Golay cell. The scan is performed so that $0^{\circ}$ corresponds to radiation emitted normal to the front facet and $180^{\circ}$ corresponds to radiation emitted normal to the rear facet, indicated in Figure 3(a). The cryostat is fitted with a custom high density polyethylene outer, allowing a full $180^{\circ}$ characterization of the emitted radiation. Figure 3(b) shows the results of these scans for successive mills and different currents. The improvement is clear, the PHA not only increases the total emitted power but also significantly improves the directionality of the emission; the directed power is up to 10 times larger than the ascleaved sample, while the total integrated power is around 3 times larger. We see the maximum power for a PHA length of $275 \mu \mathrm{m}$, while further milling showed a decrease in the emitted power because the facet reflectivity decreases below the optimum value. Another feature seen in Figure 3(b) is the asymmetry of the emission; this is particularly obvious for the longest PHA. This asymmetry may be due to small differences between the PHAs on each of the facets.

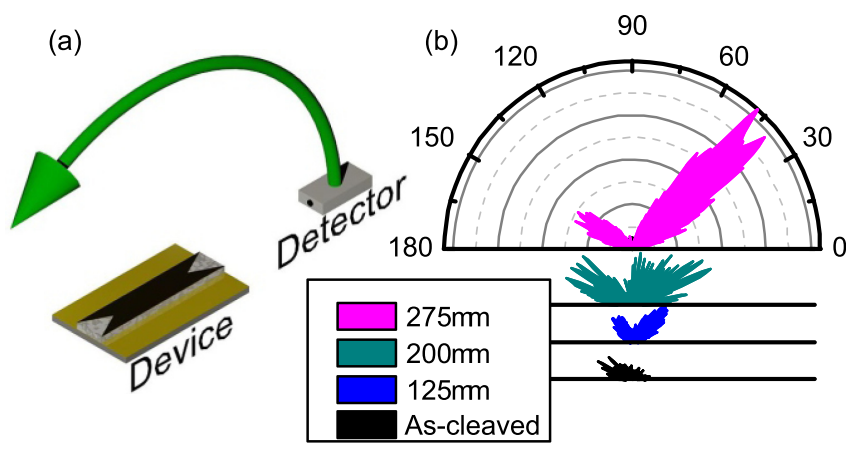

FIG. 3. (a) A schematic showing the direction of the far-field scan. (b) Far field emission plots of the same lasers as figure 2, powered in pulsed operation at $400 \mathrm{~mA}$ and plotted on the same linear scale.

Through several devices for which we have performed this procedure, we see variation in the far-field patterns observed. The far-field can also change as the current and frequency composition of the emission changes. We believe that this variability is because small changes in the near field of the device can cause large changes in the far-field emission. Such changes in the phase and frequency of the nearfield causing large variations in the far-field are well known for semiconductor lasers. ${ }^{16,17}$ In particular, we see evidence of lasing on higher order lateral modes at high drive currents in spectra from the devices, this is also associated with a change in the far-field pattern. ${ }^{18}$ Indeed, we believe that the shape of the PHA may favour high order lateral modes more than conventional MM waveguides. It may be possible to address this by changing the shape of the PHA to a tapered point rather than the "V"-shape. This variability in the exact direction of the far-field is a draw-back of this broad-band coupling method compared to the frequency selective methods based on 1-D and 2-D photonic crystals which fix the frequencies and phases of the operating mode(s).

The device shown in Figure 4 was chosen for the second part of this work because, as seen in the figure, the emission is nearly normal to the facets, simplifying the alignment and coupling for the $\mathrm{THz}$ pulse. The LI collected for the ascleaved waveguide in Figure 4(b) was collected with off-axis parabolic mirrors and collected emission from just one facet. To measure the LI for the waveguide with $200 \mu \mathrm{m}$ PHA, we collect far far-field profiles at different driving currents and integrate the measured curves to yield a measure of intensity, this provides an LI that is independent of collection angle. For the PHA device, the power begins to decrease around $500 \mathrm{~mA}$, as the device begins to mis-align.
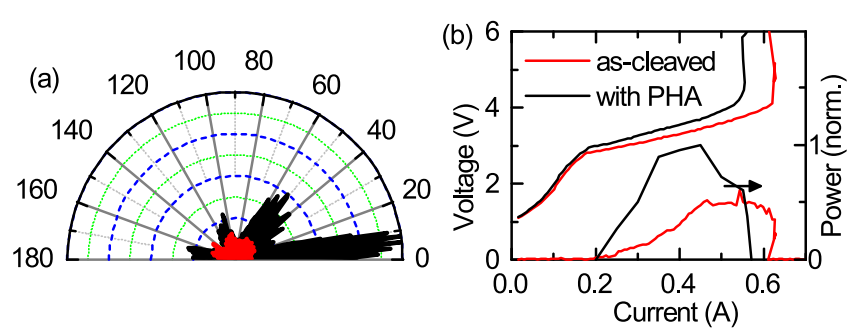

FIG. 4. Far field of device B, showing the emission pattern from the ascleaved device (red) and a device with a $200 \mu \mathrm{m}$ long "V"-shape (black). The as-cleaved LI is scaled to correct for different collection efficiencies. 
The experimental arrangement for optical seeding and coherent detection is very similar to that used in Ref. 4 and the full methods used are described there. In that and subsequent works, single plasmon QCLs were used and until now attempts to couple MM QCLs have not been successful. The experiment is based on a femtosecond laser producing $80 \mathrm{fs}$ pulses around $800 \mathrm{~nm}$. This pulse is split and one portion is used to trigger a nanosecond electrical pulse that is applied to the QCL together with a quasi-DC $10 \mathrm{kHz}$ pulse with the use of a bias-tee. A second part of the femtosecond pulse is focused onto an interdigitated photoconductive antenna and generates a single cycle $\mathrm{THz}$ pulse that is focused into the QCL cavity at the same moment that the nanosecond pulse brings the QCL above threshold. The THz pulse is focused into the QCL cavity and collected from the QCL cavity with identical f/1.67 parabolic optics. The collected QCL emission is focused onto a ZnTe electro-optic crystal co-linearly with a third portion of the femtosecond pulse, the probe. The polarization change induced in the femtosecond pulse is measured with the aid of a quarter-wave plate, a Wollaston prism, and a pair of balanced photodiodes. The signal, proportional to the $\mathrm{THz}$ field incident on the ZnTe crystal, is measured by a lock-in amplifier with a reference signal given by the quasi-DC generator at $10 \mathrm{kHz}$. By scanning the delay of the probe pulse, by the use of a mechanical delay line, we measure the signal shown in Figure 5(a). For this scan, the QCL is operated near maximum output power. Because the measurement of the QCL emission in Figure 5 is coherent, taking the Fourier transform reveals the spectrum of the emitted radiation. This is shown in Figure 5(c).

There are some notable differences between the seeding seen here for this MM device, and the type of seeding typically seen for single plasmon devices. First, we do not see a well-defined amplified THz seed pulse exit the QCL cavity, as is typically seen for single plasmon devices with the same active region. Rather, we see extended electric-field oscillations which slowly decay, but are still visible after the roundtrip time of the cavity ( $\sim 80 \mathrm{ps})$. This corresponds to a much narrower spectrum in frequency space, thought to be due to increased frequency filtering of the MM waveguide which has much higher loss for frequencies outside the laser

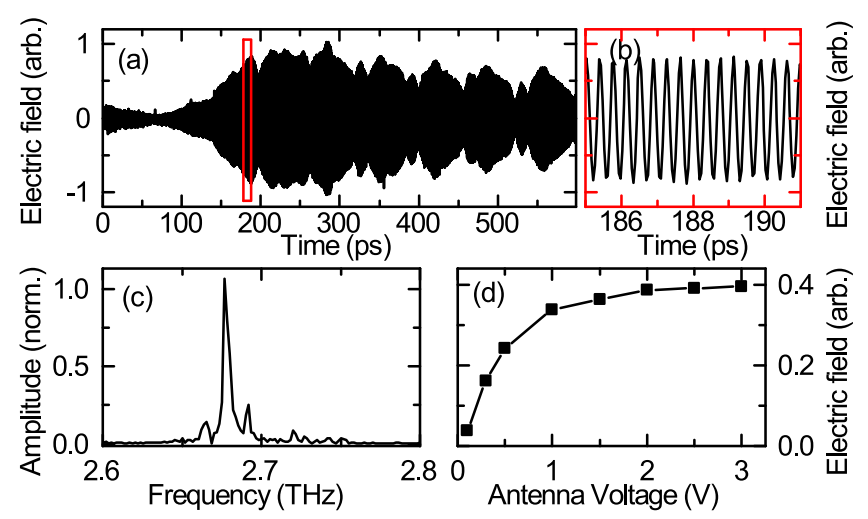

FIG. 5. Results from coherent detection of the QCL emission. (a) The timedomain electric field emitted by the QCL in the first $600 \mathrm{ps}$ after the seed pulse arrives.(b) Detail of 185-191 ps showing the electric field. (c) The Fourier transform of the time-domain electric field showing the emitted spectrum. (d) The electric field produced by the QCL at $550 \mathrm{ps}$ as a function of antenna voltage. bandwidth, compared with single plasmon waveguides. We note that Burghoff et al. ${ }^{19}$ observed well defined pulses decaying from a MM waveguide; however, the gain of that active region was particularly broad. Second, we find that the optimal delay between the nanosecond pulse, and the $\mathrm{THz}$ seed pulse tends to be 100-200 ps later than for a comparable single plasmon device. This is seen in Figure 5(a) by the decay of the THz seed pulse between 0 ps (when the seed arrives) and around $100 \mathrm{ps}$ when the nanosecond pulse arrives. This again may be due the increased frequency filtering which ensures that the seed pulse spreads more quickly to form a standing wave in the QCL cavity rather than propagate as a pulse.

Another difference between single plasmon and MM waveguides is the waveguide losses. This difference is noticeable when measuring the transmission through the waveguide when the device is in the "off" state, required as a "reference" when doing gain measurements of the type described in Ref. 15.

Finally, to verify that the QCL emission is well seeded and there is no non-synchronized component to the emission, we measure how the emission amplitude depends on the antenna voltage (linearly proportional to the $\mathrm{THz}$ seed amplitude). ${ }^{20}$ We see that as the seed amplitude is increased, the signal we measure reaches an asymptotic maximum, consistent with emission that is well synchronized with the detection system.

We have described a method to improve the output power and directionality of MM waveguides with subwavelength dimensions. The simple fabrication of this method results in monolithic devices that are easily fabricated by standard photolithographic techniques, in this work, we used a FIB for rapid prototyping. This design has the advantage that it is broadband and thus, particularly suited to optical injection seeding by broadband $\mathrm{THz}$ pulses. Further improvements could be made to the design by optimizing the tapering, so rather than using a simple "V"-shape the PHA shape was optimized for the best impedance matching between the waveguide and free-space the design could also be improved to suppress high order lateral modes. The PHA devices were used in the final part of the work to demonstrate broadband injection seeding with MM waveguides and coherent detection of the laser radiation, a measurement that has previously only been achieved with single plasmon waveguides; this opens the possibility of coherently detecting QCLs at liquid nitrogen temperatures and above.

This work was supported by the EPSRC and Agence Nationale de la Recherche (ANR, Contract No. HI-TEQ ANR-09-NANO-017). One author (J.R.F.) acknowledges funding from a Marie Curie fellowship (Grant No. 274602).

${ }^{1}$ S. Kumar and A. Lee, IEEE J. Sel. Top. Quantum Electron. 14, 333-344 (2008).

${ }^{2}$ S. Fathololoumi, E. Dupont, C. Chan, Z. Wasilewski, S. Laframboise, D. Ban, A. Mátyás, C. Jirauschek, Q. Hu, and H. C. Liu, Opt. Express 20, 3866-3876 (2012).

3"Generation and detection of terahertz radiation," in Terahertz Spectroscopy: Theory and Applications, edited by K. Peiponen, A. Zeitler, and M. Kuwata-Gonokami (Springer, 2012). 
${ }^{4}$ D. Oustinov, N. Jukam, R. Rungsawang, J. Madeo, S. Barbieri, P. Filloux, C. Sirtori, X. Marcadet, J. Tignon, and S. Dhillon, Nat. Commun. 1, 69 (2010).

${ }^{5}$ A. J. L. Adam, I. Kasalynas, J. N. Hovenier, T. O. Klaassen, J. R. Gao, E. E. Orlova, B. S. Williams, S. Kumar, Q. Hu, and J. L. Reno, Appl. Phys. Lett. 88, 151105 (2006).

${ }^{6}$ S. Kumar, B. S. Williams, Q. Qin, A. W. Lee, Q. Hu, and J. L. Reno, Opt. Express 15, 113-128 (2007).

${ }^{7}$ M. I. Amanti, M. Fischer, G. Scalari, M. Beck, and J. Faist, Nature Photon. 3, 586-590 (2009).

${ }^{8}$ Y. Chassagneux, R. Colombelli, W. Maineult, S. Barbieri, H. E. Beere, D. A. Ritchie, S. P. Khanna, E. H. Linfield, and A. G. Davies, Nature 457, 174-178 (2009).

${ }^{9}$ J. Lloyd-Hughes, G. Scalari, A. van Kolck, M. Fischer, M. Beck, and J. Faist, Opt. Express 17, 18387-18393 (2009).

${ }^{10}$ M. Amanti, M. Fischer, C. Walther, G. Scalari, and J. Faist, Electron. Lett. 43, 573-574 (2007).

${ }^{11}$ W. Maineult, P. Gellie, A. Andronico, P. Filloux, G. Leo, C. Sirtori, S. Barbieri, E. Peytavit, T. Akalin, J.-F. Lampin, H. E. Beere, and D. A. Ritchie, Appl. Phys. Lett. 93, 183508 (2008).

${ }^{12}$ M. Martl, J. Darmo, C. Deutsch, M. Brandstetter, A. M. Andrews, P. Klang, G. Strasser, and K. Unterrainer, Opt. Express 19, 733 (2011).
${ }^{13}$ The instrument used in this study is a Zeiss cross beam FIB/SEM employing $30 \mathrm{keV} \mathrm{Ga}^{+}$ions for sputtering and a Raith ELPHY Quantum digital patterning system.

${ }^{14}$ S. Barbieri, J. Alton, H. E. Beere, J. Fowler, E. H. Linfield, and D. A. Ritchie, Appl. Phys. Lett. 85, 1674-1676 (2004).

${ }^{15}$ N. Jukam, S. S. Dhillon, D. Oustinov, Z.-Y. Zhao, S. Hameau, J. Tignon, S. Barbieri, A. Vasanelli, P. Filloux, C. Sirtori, and X. Marcadet, Appl. Phys. Lett. 93, 101115 (2008).

${ }^{16} \mathrm{E}$. Kapon, J. Katz, and A. Yariv, "Supermode analysis of phase-locked arrays of semiconductor lasers," Opt. Lett. 9, 125-127 (1984).

${ }^{17}$ Y. Chassagneux, R. Colombelli, W. Maineult, S. Barbieri, S. P. Khanna, E. H. Linfield, and A. G. Davies, Appl. Phys. Lett. 96, 031104 (2010).

${ }^{18}$ P. Gellie, W. Maineult, A. Andronico, G. Leo, C. Sirtori, S. Barbieri, Y. Chassagneux, J. R. Coudevylle, R. Colombelli, S. P. Khanna, E. H. Linfield, and A. G. Davies, J. Appl. Phys. 104, 124513 (2008).

${ }^{19}$ D. Burghoff, T.-Y. Kao, D. Ban, A. W. M. Lee, Q. Hu, and J. Reno, Appl. Phys. Lett. 98, 061112 (2011).

${ }^{20}$ J. Maysonnave, N. Jukam, M. S. M. Ibrahim, R. Rungsawang, K. Maussang, J. Madéo, P. Cavalié, P. Dean, S. P. Khanna, D. P. Steenson, E. H. Linfield, A. G. Davies, S. S. Dhillon, and J. Tignon, Opt. Express 20, $16662-16670$ (2012). 